PROCEEDINGS OF THE

AMERICAN MATHEMATICAL SOCIETY

Volume 131, Number 3, Pages 845-850

S 0002-9939(02)06726-6

Article electronically published on July 2, 2002

\title{
ON THE LOCAL SPECTRAL RADIUS OF POSITIVE OPERATORS
}

\author{
MIROSŁAWA ZIMA
}

(Communicated by David R. Larson)

\begin{abstract}
We give some sufficient conditions for subadditivity and submultiplicativity of the local spectral radius of bounded positive linear operators.
\end{abstract}

\section{INTRODUCTION}

Let $A$ be a bounded linear operator in a Banach space $X$. Denote by $r(A)$ and $r(A, x), x \in X$, the spectral radius of $A$ and the local spectral radius of $A$ at $x$, respectively. Let us recall that

$$
r(A)=\lim _{n \rightarrow \infty}\left\|A^{n}\right\|^{1 / n}
$$

and

$$
r(A, x)=\limsup _{n \rightarrow \infty}\left\|A^{n} x\right\|^{1 / n} .
$$

Several interesting properties of $r(A)$ and $r(A, x)$ can be found for example in [1], 4, [5], [6], 7]. The aim of our paper is to establish some theorems on the local spectral radius of the sum and composition of two operators. The classical result states (see [5, 8]) that if $A$ and $B$ are bounded linear commutative operators, then $r(A+B) \leq r(A)+r(B)$ and $r(A B) \leq r(A) r(B)$. In 11, Daneš proved the analogous inequalities for local spectral radius. Precisely, he showed that if $A$ and $B$ are commutative, then for every $x \in X$

$$
r(A+B, x) \leq r(A, x)+r(B)
$$

and

$$
r(A B, x) \leq r(A, x) r(B) .
$$

It is easy to show that the assumption of commutativity is essential, but it can be weakened in several ways. Some theorems of this type concerning operators in partially ordered Banach spaces can be found in [2, [9], [10].

It is of interest to know whether $r(B)$ can be replaced by $r(B, x)$ in (1) and (2), that is, if it is possible to obtain the inequalities

$$
r(A+B, x) \leq r(A, x)+r(B, x)
$$

and

$$
r(A B, x) \leq r(A, x) r(B, x) .
$$

Received by the editors July 6, 2001 and, in revised form, October 15, 2001.

2000 Mathematics Subject Classification. Primary 47A11, 47B65. 
Recall that $r(A, x) \leq r(A)$ for all $x \in X, r(A)=\max \{r(A, x): x \in X\}$ and the equality $r(A)=r(A, x)$ holds on the dense subset of $X$ (see [1], [6], [7]). For the conditions ensuring $r(A)=r(A, x)$ we refer the reader to [3], [4]. Our purpose is to find conditions implying the above inequalities also in the case $r(A, x)<r(A)$ and $r(B, x)<r(B)$. It is well known that $r(A B)=r(B A)$ for any $A$ and $B$. Since $r(A B, x)$ may be different from $r(B A, x)$, we will estimate both of them.

\section{MAIN RESUlts}

For the convenience of the reader, we begin this section with some definitions.

Definition 1. A nonempty subset $K$ of a Banach space $X$ is called a cone if $K$ is closed and

(i) $\lambda x \in K$ for all $x \in K$ and $\lambda \geq 0$,

(ii) $x, y \in K$ implies $x+y \in K$,

(iii) $x,-x \in K$ implies $x=\theta$.

It is well known that $K$ induces a partial order in $X$ as follows: for $x, y \in X$ we say that $x \prec y$ if and only if $y-x \in K$ (see for example [5]).

Definition 2. A cone $K$ is said to be normal if there exists $\gamma>0$ such that if $\theta \prec x \prec y$, then $\|x\| \leq \gamma\|y\|$.

Definition 3. Let $K$ be a cone in $X$ and let $A: X \rightarrow X$ be a bounded linear operator. We say that $A$ is positive if $A(K) \subset K$.

Notice that $A(K) \subset K$ implies that $A$ is increasing, that is, if $x \prec y$, then $A x \prec A y$.

Definition 4. Let $K$ be a cone in $X, u_{0} \in K$ and $u_{0} \neq \theta$. We say that a bounded linear operator $A: X \rightarrow X$ is $u_{0}$-upper bounded if for every $x \in X$ there exists a nonnegative number $\alpha(x)$ such that $A x \prec \alpha(x) u_{0}$.

Finally, recall some properties of local spectral radius which will be useful in our further considerations (see [1]):

$$
\begin{gathered}
r(A, \lambda x)=r(A, x) \text { for all } x \in X \text { and } \lambda \neq 0, \\
r(A, x+y) \leq \max \{r(A, x), r(A, y)\} \text { for all } x, y \in X, \\
r\left(A, A^{k} x\right)=r(A, x) \text { for all } x \in X \text { and } k \in \mathbb{N} .
\end{gathered}
$$

Now, we state and prove a few theorems on subadditivity and submultiplicativity of local spectral radius of positive operators. From now on we assume that $K$ is a normal cone in a Banach space $X$ and $A$ and $B$ are bounded positive linear operators.

Theorem 1. Suppose that $A$ is $u_{0}$-upper bounded and

$$
B A^{i} B^{j} u_{0} \prec A^{i} B^{j+1} u_{0}
$$

for $i=1,2, \ldots$, and $j=0,1,2, \ldots$ Then

$$
\begin{gathered}
r\left(A+B, u_{0}\right) \leq r\left(A, u_{0}\right)+r\left(B, u_{0}\right), \\
r\left(A B, u_{0}\right) \leq r\left(A, u_{0}\right) r\left(B, u_{0}\right) \text { and } r\left(B A, u_{0}\right) \leq r\left(A, u_{0}\right) r\left(B, u_{0}\right) .
\end{gathered}
$$


Proof. The method of the proof is partly based on that used in [2 for the spectral radius. Since $A$ is $u_{0}$-upper bounded and positive, for every $x \in K$ there exists $\alpha(x) \geq 0$ such that

$$
\theta \prec A^{n} x \prec \alpha(x) A^{n-1} u_{0}
$$

for all $n \in \mathbb{N}$. Hence

$$
\left\|A^{n} x\right\| \leq \gamma \alpha(x)\left\|A^{n-1} u_{0}\right\|
$$

which gives

$$
r(A, x) \leq r\left(A, u_{0}\right)
$$

for every $x \in K$. Let $\epsilon>0$ and with $\delta_{B}=r\left(B, u_{0}\right)+\epsilon / 2$ put

$$
u=\sum_{i=0}^{\infty} \delta_{B}^{-(i+1)} B^{i} u_{0}
$$

evidently well defined. Observe that $u \in K$ and $u=\delta_{B}^{-1}\left(u_{0}+B u\right)$. Hence

$$
B u \prec \delta_{B} u
$$

and

$$
u_{0} \prec \delta_{B} u \text {. }
$$

From (12) it follows that $r\left(A, u_{0}\right) \leq r(A, u)$ which with (9) gives

$$
r(A, u)=r\left(A, u_{0}\right)
$$

Next, put $\delta_{A}=r\left(A, u_{0}\right)+\epsilon / 2$ and define

$$
v=\sum_{i=0}^{\infty} \delta_{A}^{-(i+1)} A^{i} u
$$

It is easy to see that $v \in K, u \prec \delta_{A} v, A v \prec \delta_{A} v$ and $u_{0} \prec \delta_{A} \delta_{B} v$. Moreover, by (6) and (11) we have

$$
B v=\sum_{i=0}^{\infty} \delta_{A}^{-(i+1)} B A^{i} u \prec \sum_{i=0}^{\infty} \delta_{A}^{-(i+1)} A^{i} B u \prec \sum_{i=0}^{\infty} \delta_{A}^{-(i+1)} \delta_{B} A^{i} u=\delta_{B} v .
$$

Then

$$
\begin{gathered}
(A+B) u_{0} \prec \delta_{A} \delta_{B}(A+B) v \prec \delta_{A} \delta_{B}\left(\delta_{A}+\delta_{B}\right) v \\
(A+B)^{2} u_{0} \prec \delta_{A} \delta_{B}\left(\delta_{A}+\delta_{B}\right)(A+B) v \prec \delta_{A} \delta_{B}\left(\delta_{A}+\delta_{B}\right)^{2} v
\end{gathered}
$$

and generally for all $n \in \mathbb{N}$

$$
(A+B)^{n} u_{0} \prec \delta_{A} \delta_{B}\left(\delta_{A}+\delta_{B}\right)^{n} v .
$$

Thus

$$
\left\|(A+B)^{n} u_{0}\right\| \leq \gamma \delta_{A} \delta_{B}\left(\delta_{A}+\delta_{B}\right)^{n}\|v\|
$$

and consequently

$$
r\left(A+B, u_{0}\right) \leq \delta_{A}+\delta_{B}
$$

This gives (7), since $\epsilon>0$ is arbitrary. To prove (8), observe that $A B v \prec \delta_{A} \delta_{B} v$ and $A B u_{0} \prec \delta_{A} \delta_{B} v$. Thus,

$$
(A B)^{n} u_{0} \prec\left(\delta_{A} \delta_{B}\right)^{n} v
$$


for all $n \in \mathbb{N}$. Hence

$$
\left\|(A B)^{n} u_{0}\right\| \leq \gamma\left(\delta_{A} \delta_{B}\right)^{n}\|v\|,
$$

which gives $r\left(A B, u_{0}\right) \leq \delta_{A} \delta_{B}$. The last inequality clearly implies $r\left(A B, u_{0}\right) \leq$ $r\left(A, u_{0}\right) r\left(B, u_{0}\right)$. In the same manner we can see that $(B A)^{n} u_{0} \prec\left(\delta_{A} \delta_{B}\right)^{n} v$ and in consequence (8) holds. This completes the proof of Theorem 1.

It is worth mentioning that assumption (6) is fulfilled if for example $A B=B A$ or $B A x \prec A B x$ for every $x \in K$.

Example 1. Let $X=c_{0}$ be a Banach space of all real sequences convergent to zero with supremum norm

$$
\|x\|=\sup _{i}\left|x_{i}\right| .
$$

Let $u_{0}=\left\{1 / 4^{i}\right\}$ and $K=\left\{x \in c_{0}: x=t u_{0}, t \geq 0\right\}$. Obviously, $K$ is a normal cone in $c_{0}$. For $x \in c_{0}, x=\left\{x_{i}\right\}$, consider the operators $A x=\left(x_{2}, x_{3}, x_{4}, \ldots\right)$, $B x=\frac{1}{2}\left(x_{3}, x_{4}, x_{5}, \ldots\right)$. It is easy to show that $A$ and $B$ satisfy the assumptions of Theorem 1. Particularly, $B=\frac{1}{2} A^{2}$, so (6) is fulfilled. Furthermore, if $x \in K$, then $x=t u_{0}$, where $t \geq 0$. Hence $A x=t A u_{0}=\frac{1}{4} t u_{0}$, which means that $A$ is $u_{0}$-upper bounded. Since $r\left(A, u_{0}\right)=\frac{1}{4}$ and $r\left(B, u_{0}\right)=\frac{1}{32}$, by Theorem 1 we get $r\left(A+B, u_{0}\right) \leq \frac{9}{32}$ and $r\left(A B, u_{0}\right) \leq \frac{1}{128}$. Observe that in this case we have $r(A)=1$ and $r(B)=\frac{1}{2}$.

Theorem 2. Suppose that there exists $u_{0} \in K$ such that $A u_{0} \prec r\left(A, u_{0}\right) u_{0}$ and

$$
B A^{i} B^{j} u_{0}=A^{i} B^{j+1} u_{0}
$$

for $i=1,2, \ldots$ and $j=0,1,2, \ldots$ Then (7) and (8) hold.

Proof. First, observe that $A^{n} u_{0} \prec\left[r\left(A, u_{0}\right)\right]^{n} u_{0}$ for all $n \in \mathbb{N}$. For the element $u$ defined by (10) we have

$$
A^{n} u=\sum_{i=0}^{\infty} \delta_{B}^{-(i+1)} B^{i} A^{n} u_{0} \prec\left[r\left(A, u_{0}\right)\right]^{n} u .
$$

This gives $r(A, u) \leq r\left(A, u_{0}\right)$. In consequence, $r(A, u)=r\left(A, u_{0}\right)$. The rest of the proof runs as before.

Theorem 3. Suppose that for $u_{0} \in K$, (6) is satisfied and

$$
B u_{0} \prec r\left(B, u_{0}\right) u_{0} .
$$

Then the inequalities (7) and (8) hold.

Proof. From (16), we obtain for all $i \in \mathbb{N}$

$$
B^{i} u_{0} \prec\left[r\left(B, u_{0}\right)\right]^{i} u_{0} .
$$

Therefore for $u$ defined by (10) we have

$$
u \prec \sum_{i=0}^{\infty} \delta_{B}^{-(i+1)}\left[r\left(B, u_{0}\right)\right]^{i} u_{0}=\frac{2}{\epsilon} u_{0},
$$

which gives $r(A, u) \leq r\left(A, u_{0}\right)$. We can now proceed analogously to the proof of Theorem 1. 
Example 2. Let $X=c_{0}$ and $K=\left\{x \in c_{0}: x_{i} \geq 0, i \in \mathbb{N}\right\}$. Obviously, $K$ is a normal cone in $c_{0}$. Define $A x=\left(x_{3}, x_{4}, x_{5}, \ldots\right)$ and $B x=\left(x_{2}+x_{3}, 2 x_{3}, 2 x_{4}, \ldots\right)$. For $u_{0}=\left\{1 / 3^{i}\right\}$ we have $r\left(A, u_{0}\right)=\frac{1}{9}, r\left(B, u_{0}\right)=\frac{2}{3}$. It is clear that $B u_{0} \prec r\left(B, u_{0}\right) u_{0}$. Moreover, for $i=1,2, \ldots, j=0,1, \ldots$, we get

$$
B A^{i} B^{j} u_{0}=2^{j}\left(\frac{1}{3^{j+2 i+2}}+\frac{1}{3^{j+2 i+3}}, \frac{2}{3^{j+2 i+3}}, \frac{2}{3^{j+2 i+4}}, \ldots\right)
$$

and

$$
A^{i} B^{j+1} u_{0}=2^{j+1}\left(\frac{1}{3^{j+2 i+2}}, \frac{1}{3^{j+2 i+3}}, \frac{1}{3^{j+2 i+4}}, \ldots\right) .
$$

It follows that (6) is satisfied. By Theorem $3, r\left(A+B, u_{0}\right) \leq \frac{7}{9}$ and $r\left(A B, u_{0}\right) \leq \frac{2}{27}$. Notice that $r(A)=1$ and $r(B)=2$.

Theorem 4. If (15) is satisfied and there exists $k \in \mathbb{N}$, such that

$$
B^{k+1} u_{0} \prec r\left(B, u_{0}\right) B^{k} u_{0},
$$

then (7) and (8) hold.

Proof. For $u$ defined by (10) we get from (17)

$$
\begin{aligned}
u= & \sum_{i=0}^{k} \delta_{B}^{-(i+1)} B^{i} u_{0}+\sum_{j=1}^{\infty} \delta_{B}^{-(k+j+1)} B^{k+j} u_{0} \\
& \prec \sum_{i=0}^{k} \delta_{B}^{-(i+1)} B^{i} u_{0}+\sum_{j=1}^{\infty} \delta_{B}^{-(k+j+1)}\left[r\left(B, u_{0}\right)\right]^{j} B^{k} u_{0} \\
& =\sum_{i=0}^{k} \delta_{B}^{-(i+1)} B^{i} u_{0}+\frac{2}{\epsilon} \delta_{B}^{-(k+1)} r\left(B, u_{0}\right) B^{k} u_{0} .
\end{aligned}
$$

Put

$$
v_{0}=\sum_{i=0}^{k} \delta_{B}^{-(i+1)} B^{i} u_{0}+\frac{2}{\epsilon} \delta_{B}^{-(k+1)} r\left(B, u_{0}\right) B^{k} u_{0} .
$$

Since $A^{n} B^{i} u_{0}=B^{i} A^{n} u_{0}$ for all $i, n \in \mathbb{N}$, we obtain

$$
r\left(A, B^{i} u_{0}\right) \leq r\left(A, u_{0}\right) .
$$

Thus, by (3) and (4), $r\left(A, v_{0}\right) \leq r\left(A, u_{0}\right)$. Moreover, $u \prec v_{0}$ implies $r(A, u) \leq$ $r\left(A, v_{0}\right)$. Therefore $r(A, u) \leq r\left(A, u_{0}\right)$ and the rest of the proof runs as before.

In a similar way we can prove the following result.

Theorem 5. If (6) and (17) are satisfied and $B u_{0} \prec A u_{0}$, then the inequalities (7) and (8) hold.

Proof. It suffices to observe that in view of (6) and (17) we have $u \prec v_{0}$, where $u$ and $v_{0}$ are defined by (10) and (18), respectively. Since $B u_{0} \prec A u_{0}$, we get from (6) $B^{i} u_{0} \prec A^{i} u_{0}$ for all $i \in \mathbb{N}$. Thus

$$
v_{0} \prec \sum_{i=0}^{k} \delta_{B}^{-(i+1)} A^{i} u_{0}+\frac{2}{\epsilon} \delta_{B}^{-(k+1)} r\left(B, u_{0}\right) A^{k} u_{0} .
$$

By (3)-(5), we get $r\left(A, v_{0}\right) \leq r\left(A, u_{0}\right)$. As in the proof of Theorem 4 , this gives $r(A, u) \leq r\left(A, u_{0}\right)$. The same type of analysis as that in the proof of Theorem 1 establishes the result. 
Example 3. In the space $c_{0}$ consider the cone $K=\left\{x \in c_{0}: x_{i} \geq 0, i \in \mathbb{N}\right\}$, the operators $A x=\left(x_{2}+x_{3}, x_{3}, x_{4}, \ldots\right)$ and $B x=\left(x_{3}, x_{4}, x_{5}, \ldots\right)$ and the element $u_{0}=$ $\left(0, \frac{1}{2}, \frac{1}{4}, \frac{1}{8}, \ldots\right)$. Observe that $B A x=\left(x_{4}, x_{5}, x_{6}, \ldots\right)$ and $A B x=\left(x_{4}+x_{5}, x_{5}, x_{6}, \ldots\right)$, so $B A x \prec A B x$ for every $x \in K$ which implies (6). Furthermore, $r\left(A, u_{0}\right)=\frac{1}{2}$, $r\left(B, u_{0}\right)=\frac{1}{4}, B^{2} u_{0} \prec r\left(B, u_{0}\right) B u_{0}$ and $B u_{0} \prec A u_{0}$. By Theorem $5, r\left(A+B, u_{0}\right) \leq$ $\frac{3}{4}$ and $r\left(A B, u_{0}\right) \leq \frac{1}{8}$.

Finally, observe that $u_{0}$ can be replaced in (7) by any $x \in X$ such that

$$
r\left(A+B, u_{0}\right)=r(A+B, x), \quad r\left(A, u_{0}\right)=r(A, x) \quad \text { and } \quad r\left(B, u_{0}\right)=r(B, x) .
$$

An analogous conclusion holds for (8). Particularly, by (3), the inequalities (7) and (8) hold for $x=\lambda u_{0}, \lambda \in \mathbb{R}$. We shall prove that (7) and (8) are also satisfied for the elements $u$ and $v$ defined in the proof of Theorem 1 .

Corollary. If the assumptions of Theorem 1 are satisfied, then (7) and (8) hold for $u$ and $v$ defined by (10) and (14), respectively.

Proof. From (12) it follows that $A$ is $u$-upper bounded. It is easy to check that (6) holds with $u_{0}$ replaced by $u$. Thus, by Theorem 1 , inequalities (7) and (8) hold for $u$. In the same manner we get the conclusion for $v$.

Remark. Obviously (7) and (8) are also satisfied for any partial sum of the series (10) and (14). It is clear that the similar corollaries can be derived from Theorems $2-5$. Moreover, observe that if (6) holds, then

$$
\begin{gathered}
r\left(A+B, u_{0}\right) \leq r(A)+r\left(B, u_{0}\right), \\
r\left(A B, u_{0}\right) \leq r(A) r\left(B, u_{0}\right) \text { and } r\left(B A, u_{0}\right) \leq r(A) r\left(B, u_{0}\right) .
\end{gathered}
$$

REFERENCES

1. J.Daneš, On local spectral radius, Čas. pěst. mat. 112 (1987), 177-187. MR 88j:47004

2. A.R.Esayan, On the estimation of the spectral radius of the sum of positive semicommutative operators (in Russian), Sibirsk. Math. Zh. 7 (1966), 460-464.

3. K.H.Förster and B.Nagy, On the local spectral theory of positive operators, Operator Theory: Advances and Applications, vol. 28, Birkhäuser, Basel, 1988, pp. 71-81. MR 89g:47049

4. K.H.Förster and B.Nagy, On the local spectral radius of a nonnegative element with respect to an irreducible operator, Acta Sci. Math. 55 (1991), 155-166. MR 92j:47016

5. M.A.Krasnoselskii et al., Approximate solutions of operator equations, Noordhoff, Groningen, 1972. MR 52:6515

6. K.B.Laursen and M.M.Neumann, Introduction to local spectral theory, Lond. Math. Soc. Monographs, Oxford Univ. Press, 2000. MR 2001k:47002

7. V.Müller, Local spectral radius formula for operators in Banach spaces, Czechoslovak Math. J. 38 (1988), 726-729. MR 89g:47005

8. F.Riesz and B.Sz.-Nagy, Functional analysis, Ungar, New York, 1955. MR 17:175i

9. M.Zima, A theorem on the spectral radius of the sum of two operators and its applications, Bull. Austral. Math. Soc. 48 (1993), 427-434. MR 94j:47006

10. M.Zima, On the local spectral radius in partially ordered Banach spaces, Czechoslovak Math. J. 49 (1999), 835-841. MR 2001m:47011

Institute of Mathematics, University of Rzeszów, Rejtana 16 A, 35-310 Rzeszów, POLAND

E-mail address: mzima@univ.rzeszow.pl 